\title{
Wi-Fi Network Profiling and QoS Assessment for Real Time Video Streaming
}

\author{
Dr. Jennifer S. Raj, \\ Department of ECE, \\ Gnanamani College of Technology, \\ Namakkal, India. \\ jennifer.raj@gmail.com
}

\section{Mr. C. Vijesh Joe,}

Assistant Professor,

Department of Computer Science and Engineering,

VV College of Engineering,

Tirunelveli, India.

vijesh.joe@gmail.com

\begin{abstract}
The municipal wireless networks are developed using Wi-Fi connectivity for establishing smart cities. Wi-Fi access is provided to several thousands of cities worldwide. However, the overall utilization profile, Quality of Experience (QoE) of the user and Quality of Service (QoS) analysis has not been performed in most of these cities. There are 304 free wi-fi hotspots available in the Coimbatore City. The service availability, network performance and user connection data are analyzed for a duration of one year. The 304 hotspots are categorized into North, East, West, South and Centre zones. Video streaming experiment is conducted in these zones. The data set is leveraged for establishing and verifying the correlation between the area specific events and total user access in the region.
\end{abstract}

Keywords: Event identification, Video streaming, Public Wi-Fi, Utilization profile, Quality of Service

\section{Introduction}

Along with public utilities like power and water, internet access is also provided by several cities across all continents since the last decade [1]. The community wireless network is developed for providing Wi-Fi access to the public with the evolution of smart cities. This promotes several new service creation and digital inclusion to the citizens. Public and private 
buildings in the US provided free Wi-Fi access across several cities in the United States despite the late deployment of 3G technology [2]. Low demand, high cost, technical and regulatory issues, and various other factors caused most of these services to be discontinued by the end of the decade. The most suitable business models for effective operation and deployment of wireless network technology based on their pros and cons are discussed widely among researchers and the municipality [3].

Smart city and urban planning is integrating the provision of public Wi-Fi coverage in recent days [4]. The overall urban development plan of China has integrated free Wi-Fi network coverage for around 300 municipalities towards transforming into smart cities. Various forms of Wi-Fi access is provided by thousands of cities worldwide. Curitiba (Brazil), New York, Seoul, Paris, Adelaide, Buenos Aires and Barcelona are some of the example cities with largest networks consisting of a large number of hotspots [5-7]. However, the amount of data or connection time is limited by most cities or free data access is not provided. Irrespective of the scope of service and type, the analysis of overall utilization profile, user Quality of Experience (QoE) and Quality of Service (QoS) of the network are not estimated in most cities [8].

The city Corporation of Coimbatore in Tamil Nadu, India earmarked a budget of Rs.1.5 Crores for the introduction of free Wi-Fi in the year 2015. Since then, the Wi-Fi zones has increased to 304 in the year 2021 [6]. The five regions of the city namely Central, West, East, South and North are covered by these Wi-Fi zones providing easily accessible free of charge internet access to the citizens. A public bidding process is used for identifying the internet service provider. IEEE 802.11n, 803.11g, 802.11b and 802.11a Wi-Fi modes are supported in these zones. 70\% area of the region is to be covered by the ISP based on the contract [9]. The performance of these Wi-Fi networks are analyzed using certain test user samples from randomly chosen 10 zones across different regions of the city. The two main goals of this research are to evaluate the user QoE and network QoS for video at data streaming purposes. Further, evaluation of feasibility of accessing Wi-Fi data and identifying specific events and happenings of the area are performed. This model can be applied to other major cities and large Wi-Fi networks.

\section{Background and Problem Formulation}

The service availability, network performance and user connection related information is gathered over a duration of one year from test user samples from randomly chosen 10 zones 
across different regions of the city [10]. Three major hypothesis are validated with the help of this research. The service to the users may be denied unnecessarily based deductive estimates during static admission control. This diminishes the benefits of the user [11]. The users may not be able to access their preferred applications due to the average per-user throughputs for traffic shaping. People crowding in public areas may be identified using the number of Wi-Fi users. The service performance may be degraded when a new connection is blocked due to admission control [12]. The service providers make use of static admission control despite the significant advancements in the wireless network admission control. Poor user experience may be avoided by dynamic measurement-based admission control. This in turn reduces the negative impacts on QoE and QoS due to increase in the number of users overcoming the drawbacks of static admission control [9].

The general network performance degradation is avoided by ensuring maximum bandwidth by packet queuing with the help of traffic shaping technique [13]. When the network is not utilized completely, video streaming and other high bandwidth demanding services may be accessed by the user through traffic shaping by huge public Wi-Fi networks. The success of this public policy also depends on blocking the users from accessing certain services with the help of strict and static system resource utilization control [14]. Single users trying to stream videos on Wi-Fi face considerable variation in the QoE perception. Certain regions offer data rate over $512 \mathrm{Kbps}$ while in certain regions, the data rate is considerably low. $1200 \mathrm{Kbps}$ coding rate is used for streaming videos when the network is not utilized completely. The user experience is affected negatively by video interruptions. This factor is used for analyzing the QoE factor [7]. When there is a scarcity of network resources, maximum per-user bandwidth is allocated using dynamic traffic shaping. In public places, the crowd can be identified based on the number of users linked with the public Wi-Fi network in any specific location. The number of users are compared across different time duration to check for correlation when generic events occur, causing people gather at multiple locations simultaneously [15]. However, in such cases, the number of Wi-Fi users are diluted as people spread over multiple locations. Using this correlation, unexpected traffic jams and other unpredictable events can be identified early based on variation in Wi-Fi hotspot utilization due to people crowding in the region. Figure 1 represents the various Wi-Fi hotspots in Coimbatore Region. 


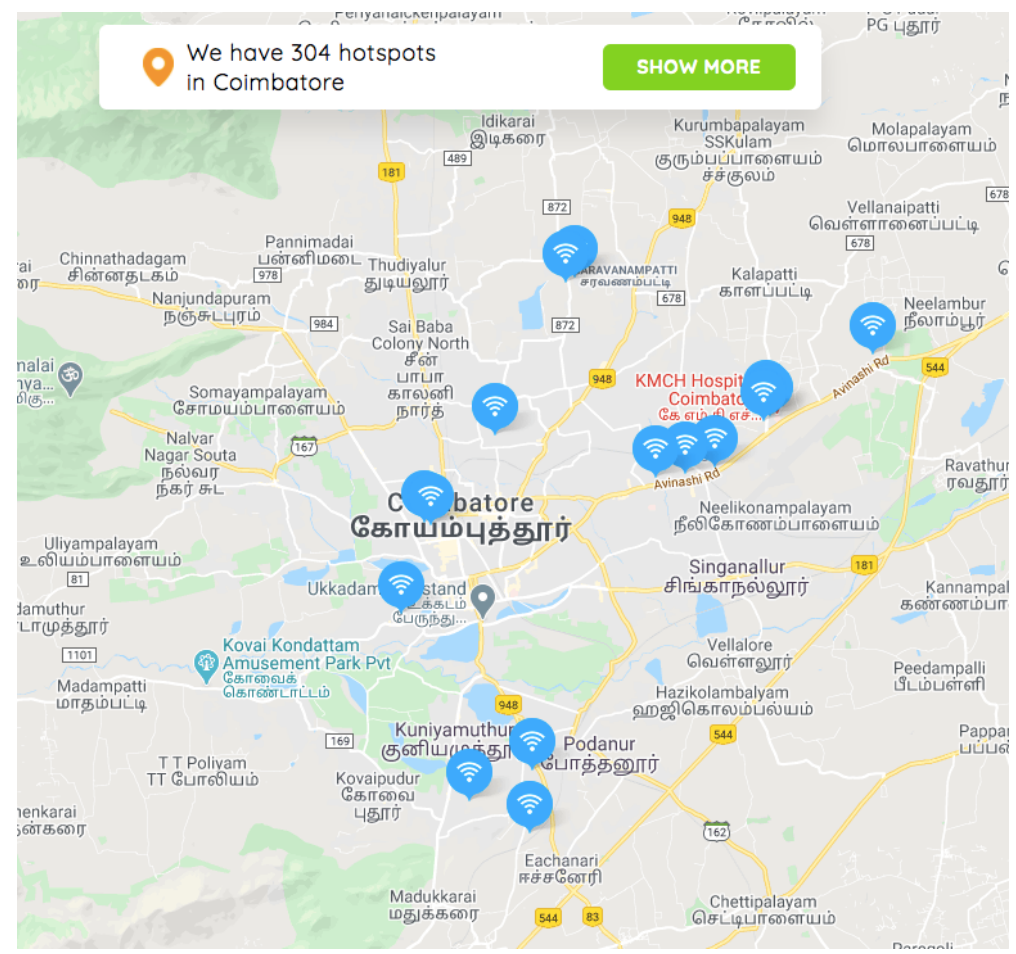

Figure 1: Wi-Fi hotspots in Coimbatore Region

\section{Proposed Research Methodology}

Event identification, video streaming and network usage schemes are the major categories under which the performance of hotspots are analyzed based on profiling user behavior. 10 events, 5 videos and 15 utilizations are demonstrated for analysis at 10 randomly selected hotspots across the city. The hotspots are numbered from 1 to 10 for easy referencing. Two hotspots from each region is chosen for comparing the various levels of users. Logistics, safety and utilization criteria are measured on spot for video streaming. Significant events hosted around the selected hotspots are considered for event identification [4]. This gives an overview of the status across the North, East, West, South and Centre zones. The obtained values with predetermined maximum users are estimated and tabulated. This also helps in analyzing the number of people who visit specific locations in the city corporation. Provisioning wired and wireless networks in these locations can be performed based on the information obtained from these values along with the admission control policies. Active as well as passive measurement schemes are used for estimating the performance, availability and accessibility of network utilization. QoE and performance is affected by the total users accessing the Wi-Fi point in the specific region. Data is collected over a duration of one year. Incoming bitrate and number of users connected to the network are managed by ISPs [3]. The 
packet loss, latency and throughput are calculated on an hourly basis by transmitting a packet sequence. Service utilization and number of users are dependent on the access provided. In video streaming, analysis is performed based on logistics, safety and utilization. QoS and QoE metrics are based on the received bit rate, number and duration of interruptions, as well as the playback start time.

\section{Results and Discussion}

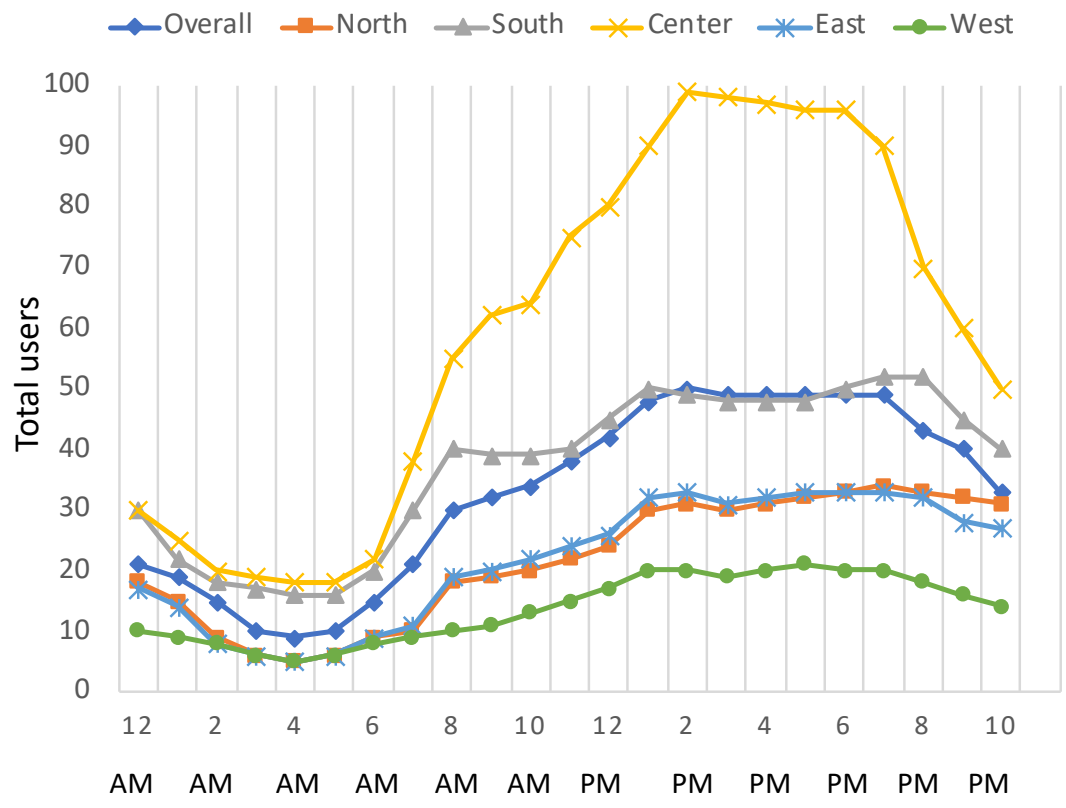

Figure 2: Hourly average user count

The results obtained from this experimentation is categorized into three segments and analyzed. The network utilization and user access are estimated based on the number of users accessing each hotspot at specific time durations during the day. The peak, average and minimal service utilization duration is analyzed. Except certain special conditions, the number of users in each zone is less on an average. This behavior is observed in most of the Wi-Fi nodes. The nodes installed near residential buildings has large number of users throughout the day. The regions are analyzed for the usage patterns based on the hours and days of usage, seasonality and location inclusive of religious, stopover, passage, leisure and sports. During business hours and weekdays, certain regions have more activity whereas in other regions, the activity is more during weekends and evenings. Based on these values, the category of region, traffic shaping policies and various admission control schemes are configured to improve the 
capabilities of network management. Figure 2 provides the hourly average user count and figure 3 provides the total number of users across randomly chosen nodes in the network.

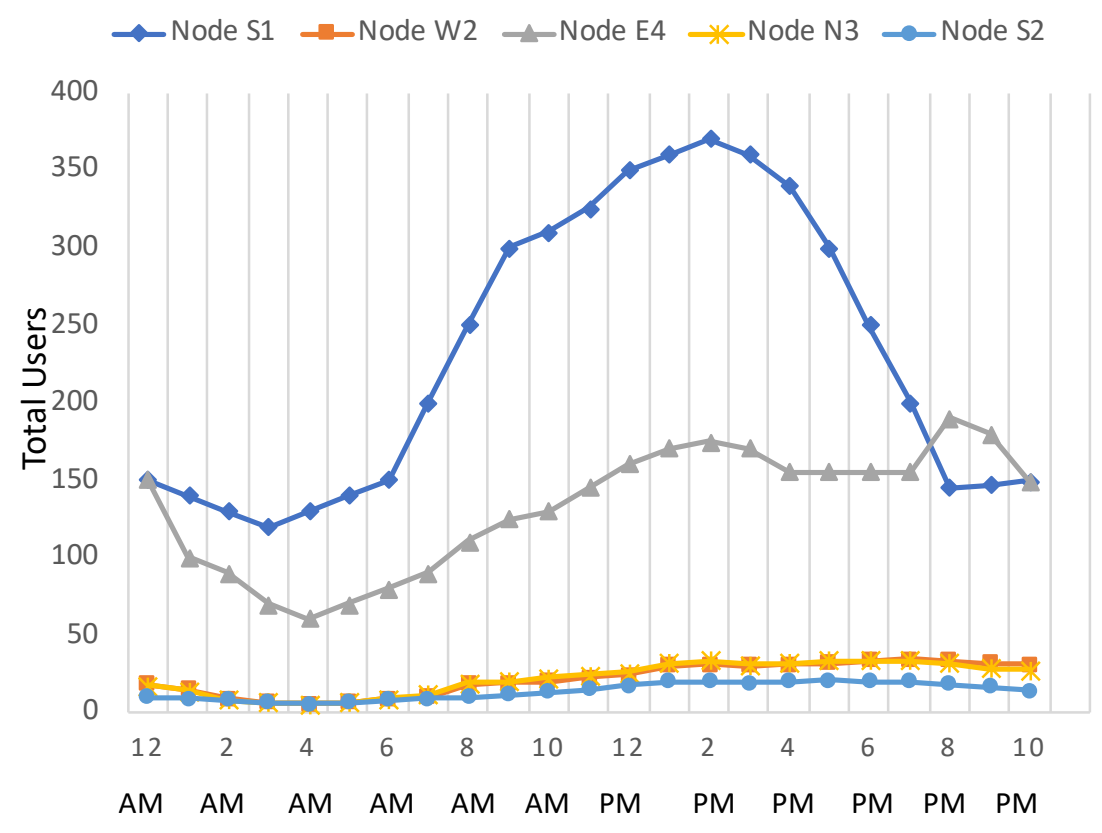

Figure 3: Total users randomly chosen nodes

Based on the network capacity provisioning, enforcement of admission control enables users to connect when the maximum users in the network exceeds the predetermined capacity of the network. Service degradation may be prevented by enforcing admission control. When the number of users exceeds the static capacity of the system, real-time monitoring is performed over the system capacity. Service degradation occurs under such scenarios. The public policy is affected when the admission control prevents users from connecting to the network prior to the network capacity reaching its maximum level. Performance degradation also occurs when the user is blocked from connecting to the network by the system. Wi-Fi based internet access provision is largely based on the availability factor as the service quality based first impression of the user is defined by this factor. However, despite the high availability of the ISP, user quality of experience is significantly affected by the service availability. 


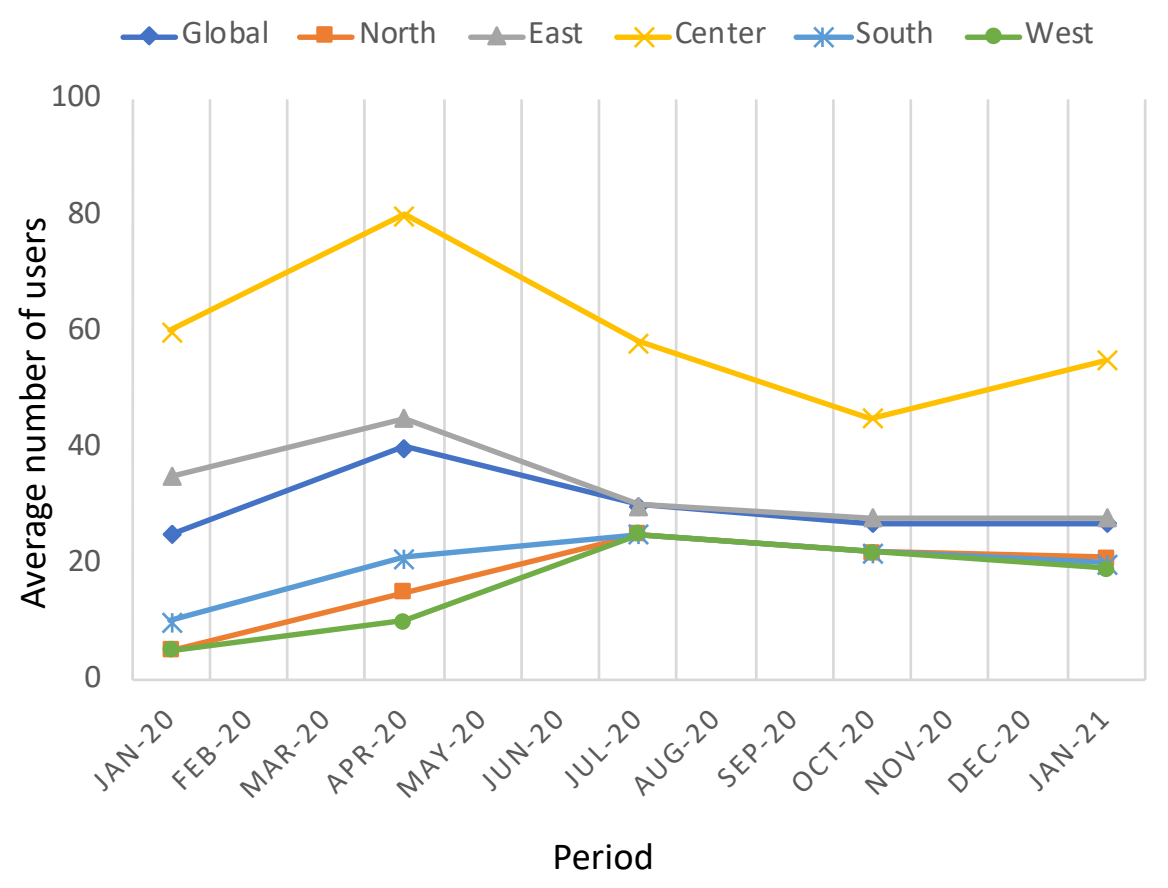

Figure 4: Average number of users

Figure 4 provides the average number of users across various nodes in the network. In order to improve the features for the providing free Internet to the citizens, several valuable inferences were made out of this study. High packet loss, high delay and low throughput affects the performance of the wired networks. The network usage and data rate influences the measurements of video streaming. Even small number of users may affect the performance of the network in a significant manner. Similar behavior can be noticed during different periods ruling out the possibility of Wi-Fi misconfiguration. Meticulous management of network infrastructure is essential during implementation of the city corporation wireless networks at both wireless and wired levels. Even while outsourcing the operation to a private ISP, this inference is valid. Figure 5 provides the cumulative throughput of the data utilization across the network. 


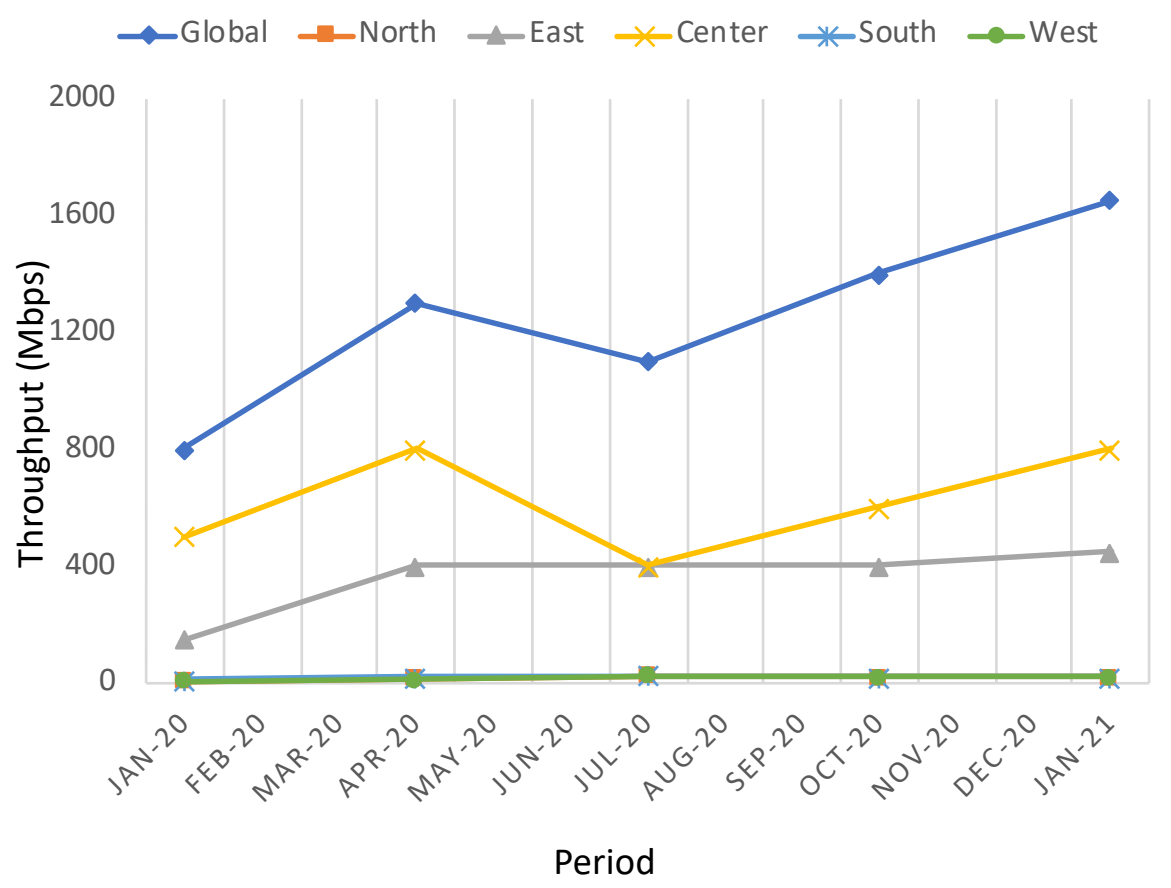

Figure 5: Cumulative throughput

The ISP backbone network does not consist of management data, configuration, technology and topology linking the Wi-Fi nodes to the different regions. Due to this, verification of the Wi-Fi network access performance is challenging. The wireless and wired networks need higher control levels despite the specified recommendations. For enforcing high quality service, monitoring and implementing end-to-end QoE and QoS is insufficient. The wired network characteristics may be understood with the help of superior measurement tools. The wired ISP network operation and quality provisioning is not impacted by the path rate and path load.

\section{Conclusion}

Since the year 2015, the Coimbatore city corporation has been providing free Wi-Fi access to its citizens at multiple locations. Currently, this scheme is in operation at 304 locations across the city. A comprehensive study is performed in this paper analyzing data over a duration of 1 year. Factors assessed includes identification of occurrence of events based on the number of users connected to the Wi-Fi network, Wi-Fi video streaming QoE across the five regions of the city and QoS parameters to estimate the Wi-Fi performance. The inferences made from this study include identification of generic events and other significant occurrences 
based on the crowd with the help of the number of users linked to the Wi-Fi network in the location, video streaming with higher bandwidth without the use of traffic shaping when the network is underutilized and utilization of priori estimates for using no admission control to approve several users. There is a significant scarcity of literature that studies these wireless networks at a large scale over a longer duration of time. Event identification, video quality and user access factors are analyzed in this paper due to the size and scope limitations. Machine learning, time series forecast and various other schemes may also be used for automatic identification of event and dynamic access control which may be addressed in future work. Rescue, healthcare, law enforcement and traffic control departments may be benefitted by foreseeing and detecting unplanned events like undetected demonstrations or sudden traffic jams that occur in these Wi-Fi zones.

\section{References}

[1] Brito, I. V. S., \& Figueiredo, G. B. (2017). Improving QoS and QoE through seamless handoff in software-defined IEEE 802.11 mesh networks. IEEE Communications Letters, 21(11), 2484-2487.

[2] Ali, J., Ahmad, R. W., Maqsood, T., Rodrigues, J. J., Ul Haq, N., Sarwar, S., ... \& Madani, S. A. (2018). Network selection in heterogeneous access networks simultaneously satisfying user profile and QoS. International Journal of Communication Systems, 31(13), e3730.

[3] Mohammadani, K. H., Butt, R. A., Memon, K. A., Pirzado, A. A., Faheem, M., Abro, A., ... \& Ain, N. U. (2021). A QoS provisioning architecture of fiber wireless network based on XGPON and IEEE 802.11 ac. Journal of Optical Communications, 1(ahead-of-print).

[4] de C Neto, J. M., G Neto, S. F., de Santana, P. M., \& de Sousa, V. A. (2020). Multi-cell LTE-U/Wi-Fi coexistence evaluation using a reinforcement learning framework. Sensors, 20(7), 1855.

[5] Ishikawa, N., Ohishi, Y., \& Maeda, K. (2019). Nulls in the air: Passive and lowcomplexity QoS estimation method for a large-scale Wi-Fi network based on null function data frames. IEEE Access, 7, 28581-28591.

[6] Miraz, M. H., Molvi, S. A., Ganie, M. A., Ali, M., \& Hussein, A. H. (2017). Simulation and analysis of quality of service (QoS) parameters of voice over IP (VoIP) traffic through heterogeneous networks. arXiv preprint arXiv:1708.01572. 
[7] Lin, Y. D., Atov, I., \& Pescape, A. (2018). Network testing and analytics. IEEE communications magazine, 56(3), 170-170.

[8] Khiat, A., Bahnasse, A., El Khaili, M., \& Bakkoury, J. (2017). Wi-Fi and WiMax QoS Performance Analysis on High-Level-Traffic using OPNET Modeler. Pertanika Journal of Science \& Technology, 25(4).

[9] Moura, H., Nunes, M., Miranda, G., Macedo, D. F., \& Correia, L. H. (2019, September). Estimating Quality of Service on Wi-Fi Stations Using Recurrent Neural Networks. In 2019 IEEE 30th Annual International Symposium on Personal, Indoor and Mobile Radio Communications (PIMRC) (pp. 1-6). IEEE.

[10] Obazee, N. J. (2020). THROUGHPUT IMPROVEMENT AND COMPARATIVE PERFORMANCE ANALYSIS OF INTEGRATED NETWORKS.

[11] Gacanin, H., \& Ligata, A. (2017). Wi-Fi self-organizing networks: Challenges and use cases. IEEE Communications Magazine, 55(7), 158-164.

[12] Toldinas, J., Lozinskis, B., Baranauskas, E., \& Dobrovolskis, A. (2019, June). MQTT Quality of Service versus Energy Consumption. In 2019 23rd International Conference Electronics (pp. 1-4). IEEE.

[13] Fauzi, R. U. A. (2019). The Role Of Free Wireless Fidelity (Wi-Fi) Service, Food Quality, And Servicescape Toward Customer Satisfaction. Asian journal of social science research, 2(2).

[14] Chen, J. I. Z., \& Smys, S. (2020). Social Multimedia Security and Suspicious Activity Detection in SDN using Hybrid Deep Learning Technique. Journal of Information Technology, 2(02), 108-115.

[15] Raj, J. S. (2019). QoS optimization of energy efficient routing in IoT wireless sensor networks. Journal of ISMAC, 1(01), 12-23. 